\title{
Serum bicarbonate and dehydration severity in gastroenteritis
}

\author{
Hassib Narchi
}

\begin{abstract}
The concentration of bicarbonate was measured in serum samples from 106 children with gastroenteritis and dehydration. A concentration less than 22 mmol/1 was more common in children with severe dehydration, but the magnitude of bicarbonate reduction was not significantly different with increasing degrees of dehydration. Doctors should not rely on the serum bicarbonate concentration when assessing fluid deficit. (Arch Dis Child 1998;78:70-71)
\end{abstract}

Keywords: dehydration; gastroenteritis; serum bicarbonate

Metabolic acidosis occurs in dehydrated patients with gastroenteritis; there are multiple causes of this acidosis. ${ }^{1-5}$ It is generally believed that acidosis, equated with a reduced concentration of bicarbonate in serum, reflects the severity of dehydration, although no study substantiating this has been found. Inappropriate hydration may occur if the correlation between low serum bicarbonate concentrations and dehydration is unfounded. This paper reports a prospective study to assess whether such a correlation exists.

\section{A1-Hasa Specialty Services Division, Saudi \\ Aramco-A1-Hasa \\ Health Center, Saudi \\ Aramco Medical \\ Services Organization, \\ Box 6030, Mubarraz \\ 31311, Kingdom of \\ Saudi Arabia \\ Correspondence to: \\ Dr Narchi.}

Accepted 11 August 1997

\section{Methods}

Over a four month period, all children presenting to hospital with gastroenteritis and dehydration were studied. The inclusion criteria were: age 1 month to 5 years; diarrhoea (three or more watery stools) or vomiting for less than 72 hours at presentation; and dehydration. The exclusion criteria were: extraintestinal infection; renal, respiratory, cardiac, neurological, or endocrine anomalies; chronic diarrhoea; malnutrition; failure to thrive; and administration of diuretics or intravenous fluids within one week of presentation.

Table 1 Clinical assessment of severity of dehydration

\begin{tabular}{llll}
\hline & \multicolumn{2}{l}{ Severity of dehydration } & \\
\cline { 2 - 4 } & Mild & Moderate & Severe \\
\hline $\begin{array}{lll}\text { Estimated body weight loss } \\
(\%)\end{array}$ & 5 & & \\
Skin turgor & Slightly decreased & Decreased & Very decreased \\
Oral mucosa & Dry & Very dry & Parched \\
Eyes & Normal & Sunken & Grossly sunken \\
Tears & \pm Decreased & Absent & Absent \\
Fontanelle & Normal & Depressed & Sunken \\
Heart rate & \pm Increased & Increased & Marked tachycardia \\
Pulses & Normal strength & Weak & Feeble or impalpable \\
Blood pressure & Normal & Decreased & Decreased \\
Respiration & Normal & Deep, may be rapid & $\begin{array}{l}\text { Deep and rapid } \\
\text { Orine output }\end{array}$ \\
Level of consciousness & Mild oliguria & Oliguria & Oliguria-anuria \\
& Irritable & Lethargic & Unresponsive \\
\hline
\end{tabular}

The degree of dehydration was estimated as mild, moderate, or severe by a paediatrician or an emergency room doctor (table 1). Concentrations of urea, creatinine, electrolytes, and bicarbonate in serum were measured on a venous specimen before treatment. A reduced serum bicarbonate concentration was defined as less than $22 \mathrm{mmol} / \mathrm{l}$ and an increased anion gap as greater than $16 \mathrm{mmol} / \mathrm{l}$. Blood $\mathrm{pH}$ was not routinely measured. The glomerular filtration rate (GFR) was estimated by the formula devised by Schwartz et al. ${ }^{6}$ It was defined as reduced if it fell two standard deviations below the mean for age and sex. The specific gravity and $\mathrm{pH}$ of urine were measured on the first urine sample obtained at presentation. Rehydration treatment (by mouth or intravenous) was prescribed at the discretion of the attending doctor.

Statistical analysis of the results was carried out by the Mann-Whitney test to compare two means, analysis of variance for more than two means, the Kruskal-Wallis one way analysis of variance for non-parametric means, and the $\chi^{2}$ test for proportions.

\section{Results}

One hundred and six children were studied (table 2). Sixteen had isolated diarrhoea and 17 isolated vomiting. Six had bacterial enteritis (salmonella or shigella infection) and the remainder were assumed to have a viral infection. Dehydration was hyponatraemic in four children, hypernatraemic in three, and isonatraemic in the remainder. Ninety eight patients required intravenous hydration.

There was no significant difference in age, sex, serum sodium, potassium, or urea, anion gap, urine $\mathrm{pH}$, or urine specific gravity between the three groups of children with dehydration (table 2). The GFR was measured in 71 patients and was reduced in 12 , but no case of hyperkalaemia was observed.

Reduced serum bicarbonate concentrations occurred in 72 patients (68\%), with a significant difference between the three groups $(p=$ 0.009). When the concentration of bicarbonate was studied by age group and the degree of dehydration (table 2), it was found to be overall significantly lower in the younger age groups $(\mathrm{p}=0.01)$, and particularly with mild ( $\mathrm{p}=$ $0.02)$ but not moderate $(\mathrm{p}=0.08)$ nor severe dehydration $(p=0.25)$. Within each age group the difference in bicarbonate concentration with varying degrees of dehydration did not reach statistical significance $(\mathrm{p}=0.8,0.5$, and $0.4)$. Although reduced bicarbonate concentrations were always present in children with severe dehydration, the proportion of children 
Table 2 Clinical characteristics of dehydrated patients

\begin{tabular}{|c|c|c|c|c|c|c|c|c|c|}
\hline \multirow[b]{2}{*}{ Mean (SD) age (months) } & \multicolumn{2}{|c|}{ Mild $(n=67)$} & \multicolumn{2}{|c|}{ Moderate $(n=29)$} & \multicolumn{2}{|c|}{ Severe $(n=10)$} & \multicolumn{2}{|c|}{ Total $(n=106)$} & \multirow{2}{*}{$\frac{p \text { Value }}{0.6}$} \\
\hline & 22.6 & $(15.7)$ & 22.7 & $(16)$ & 27.0 & $(16.8)$ & 23.1 & $(15.8)$ & \\
\hline No of boys & 33 & & 14 & & 7 & & 54 & & 0.44 \\
\hline No with diarrhoea & 53 & & 26 & & 10 & & 89 & & 0.15 \\
\hline No with vomiting & 55 & & 27 & & 8 & & 90 & & 0.34 \\
\hline \multicolumn{10}{|l|}{ Mean (SD) bicarbonate concentration } \\
\hline$(\mathrm{mmol} / \mathrm{l})$ & 19.5 & $(3.9)$ & 19.1 & $(3.4)$ & 16.9 & (3.8) & 19.1 & $(3.8)$ & 0.7 \\
\hline Age $<1$ year $(n=36)$ & 18.4 & $(4.4)(n=23)$ & 18.9 & $(3.9)(n=10)$ & 14.0 & $(3.6)(n=3)$ & 18.1 & $(4.3)$ & 0.8 \\
\hline Age $1-2$ years $(n=28)$ & 18.7 & $(3.3)(n=18)$ & 17.4 & $(3.3)(n=9)$ & 20.0 & $(0)(n=1)$ & 18.3 & $(3.2)$ & 0.5 \\
\hline Age $2-5$ years $(n=42)$ & 21.0 & $(3.4)(n=26)$ & 20.9 & $(2.2)(\mathrm{n}=10)$ & 17.8 & $(3.6)(n=6)$ & 20.5 & (3.3) & 0.4 \\
\hline $\mathrm{p}$ Value & 0.02 & & 0.08 & & 0.25 & & 0.01 & & \\
\hline No with bicarbonate $<22 \mathrm{mmol} / 1$ & 39 & & 23 & & 10 & & 72 & & 0.009 \\
\hline No with bicarbonate $<16 \mathrm{mmol} / 1$ & 11 & & 6 & & 4 & & 21 & & 0.2 \\
\hline Mean (SD) anion gap (mmol/l) & 16.7 & (3.9) & 17.8 & $(4.1)$ & 17.2 & $(4.7)$ & 17.1 & (4) & 0.5 \\
\hline No with anion gap $>16 \mathrm{mmol} / 1$ & 39 & & 16 & & 5 & & 60 & & 0.87 \\
\hline Mean $(\mathrm{SD})$ potassium $(\mathrm{mmol} / \mathrm{l})$ & 4 & $(0.5)$ & 4.1 & $(0.6)$ & 3.4 & $(0.5)$ & 3.9 & $(0.6)$ & 0.2 \\
\hline Mean (SD) GFR $\left(\mathrm{ml} / \mathrm{min} / 1.73 \mathrm{~m}^{2}\right)$ & 98.6 & (27) & 96.4 & (17) & 87.5 & (39) & 96.3 & (26) & 0.7 \\
\hline Proportion with reduced GFR (\%) & \multicolumn{2}{|c|}{10.5} & \multicolumn{2}{|c|}{17.3} & \multicolumn{2}{|l|}{40} & \multicolumn{2}{|l|}{16.9} & 0.08 \\
\hline Mean urine specific gravity & \multicolumn{2}{|c|}{1.014} & \multicolumn{2}{|c|}{1.020} & \multicolumn{2}{|c|}{1.020} & \multicolumn{2}{|c|}{1.017} & 0.16 \\
\hline Mean (SD) urine $\mathrm{pH}$ & 5.7 & (1) & 6.1 & (1) & 5.2 & $(0.4)$ & 5.7 & $(0.9)$ & 0.24 \\
\hline
\end{tabular}

with concentrations less than $16 \mathrm{mmol} / 1$ was not significantly different between the three groups $(p=0.3)$.

The GFR was reduced in 52 patients with bicarbonate concentrations less than 22 $\mathrm{mmol} / \mathrm{l}$. There was no statistical difference in the bicarbonate concentration nor the anion gap between the 40 patients with a normal and the 12 with a reduced GFR ( $\mathrm{p}=0.7$ and 0.5 , respectively).

The 16 patients who had isolated diarrhoea were compared with the 73 patients who also had vomiting. There was no difference in the mean bicarbonate concentration (18.3 $v 19.6$ $\mathrm{mmol} / \mathrm{l}, \mathrm{p}=0.09)$ nor in the anion gap (16.1 v $17.7 \mathrm{mmol} / \mathrm{l}, \mathrm{p}=0.5)$.

\section{Discussion}

Reduced serum $\mathrm{pH}$ defines acidosis. In most cases of gastroenteritis with dehydration, however, serum electrolytes are measured, but not $\mathrm{pH}$. Doctors often equate metabolic acidosis with a reduction in serum bicarbonate, which was present in $68 \%$ of our patients. It is possible that some of these patients might have had a normal blood $\mathrm{pH}$ with associated respiratory alkalosis. This is, however, unlikely, as none had a respiratory problem. Although vomiting induces gastric losses of acidic ions, there was no difference in serum bicarbonate concentrations between patients with and without vomiting. It is therefore likely that the reduction in serum bicarbonate concentration represents a true metabolic acidosis.

Although reduced bicarbonate concentrations were more frequent with increasing degrees of dehydration, the magnitude of reduction was not significantly different with increasing severity of dehydration. With no difference in the bicarbonate concentrations in patients with normal or reduced GFR, a reduction of renal function with dehydration does not explain the acidosis, nor does urinary bicarbonate loss, as the urine $\mathrm{pH}$ did not differ between the three groups. ${ }^{145}$ A normal urine $\mathrm{pH}$ cannot exclude all distal tubular acidification defects, however. A role for hypokalaemia in renal tubular function is also unlikely as there was no significant difference in serum potassium concentrations. With no difference in the anion gap, tissue hypoperfusion with lactic acidosis ${ }^{5}$ is also an unlikely factor, although lactic acid was not measured. Acidosis with an increased anion gap has been found more often in infants with prolonged diarrhoea and underlying malnutrition, ${ }^{2}$ but this study excluded these children. Acidosis is therefore most likely to be due to bicarbonate losses in diarrhoeic stools, ${ }^{34}$ the estimation of which is difficult and was not carried out in this study.

In conclusion, it is imprudent to predict the severity of dehydration and fluid requirements from bicarbonate concentrations alone as the decrease in bicarbonate concentration does not reflect the severity of fluid deficit.

The author acknowledges the use of the facilities of the Saudi Aramco Medical Services Organization for collecting the data reported in this paper. The author was employed by Saudi Aramco during the study and while the paper was being written.

1 Howland J, Marriott WM. Acidosis occurring with diarrhea. Am 7 Dis Child 1916;11:309-25.

2 Weizman Z, Houri S, Ben-Ezer G. Type of acidosis and clinical outcome in infantile gastroenteritis. $f$ Pediatr Gastroenterol Nutr 1992;14:187-91.

3 Anonymous. Diarrhoea and acid-base disturbances. Lancet 1966;i:1305-6.

4 Teree TM, Font EM, Ortiz A, Wallace WM. Stool loss and acidosis in diarrheal disease of infancy. Pediatrics 1965;36: 704-13.

5 Wang F, Butler T, Rabbani GH, Jones PK. The acidosis of cholera: contributions of hyperproteinemia, lactic acidemia, and hyperphosphatemia to an increased serum anion gap. N Engl f Med 1986;315:1591-5.

6 Schwartz GJ, Brion PL, Spitzer A. The use of plasma creatinine concentration for estimating glomerular filtration rate in infants, children, and adolescents. Pediatr Clin North Am $1987 \cdot 34: 571-90$ 